\title{
Cooperation via wireless network coding
}

\author{
DU Bing \\ Department of Electronic \\ Engineering \\ Tsinghua Univ. \\ Beijing, China \\ ice.dudu@yahoo.com.cn
}

\author{
ZHANG Jun \\ School of Electronics and \\ Information Engineering \\ Beihang Univ. \\ Beijing, China \\ buaazhangjun@vip.sina.com
}

\author{
LU JiangHua \\ Department of Electronic \\ Engineering \\ Tsinghua Univ. \\ Beijing, China \\ lujh@wmc.ee.tsinghua.edu.cn
}

\begin{abstract}
Recently, the network coding in cooperative communications has been categorized into two mainstreams, Digital Network Coding (DNC) and Analog Network Coding (ANC), which we both discuss in the article. First a brief survey of these two sub-branches is given. Then, to the very wireless characteristics fading and interference, DNC and ANC network coding way were proposed respectively, which aims at combating fading and embracing interference in wireless medium. Specifically, the idea of network-coded cooperative protocol in this paper is to combine different source nodes' information at the bit-level and signal-level corresponding to DNC and ANC in the relay node and then perform joint decoding in the destination node. The proposed DNC and ANC are demonstrated to obtain both capacity and reliability gains.
\end{abstract}

Keywords-Cooperative Communications; Digital Network Coding; Analog Network Coding; Relaying; Multiple Access Relay Channel(MARC)

\section{INTRODUCTION}

The foreseen wireless knowledge society is expected to be a highly connected (global) network where virtually any entity (man or machine) can be wirelessly connected with each other [1]. The demand for ubiquitous communications motivates the cooperation between all the entities involved in the communications, especially in the heterogeneous network, nodes or the services and operations. Hence, future wireless communications yearn for the intelligent collaborative technologies that accommodate varieties of the global wireless network. Cooperation can be seen as the action of obtaining some advantage by giving, sharing or allowing something. In nature cooperation can take place at a small scale (i.e., few entities collaborate) or large scale (i.e., massive collaboration). A similar classification holds in the wireless domain. A few nodes (e.g., terminals, base stations) can cooperate to achieve certain goals in terms of power and bandwidth.

Convergence and heterogeneous are two prominent characteristics in the next generation network. The heterogeneous networks, including Cellular Mobile Communications, Satellite Communications, GPRS, WLAN, MANET, Wi-Fi and Wireless Sensor Networks, require integrating to one convergence platform, in spite of their differences from link access protocols to resource management principles. In detail, it is difficult to detect the mobile node's position and perform fast handover due to the variable data rate, frequency, QoS, security, cost and services. All of these challenge to the mobility management technologies when users need to handover from one to another service provider. The cooperation in this field lies in the convergence of the heterogeneous network to ensure the consistent communication services and choose a best available network to access when multiple systems are involved.

Within a random homogeneous wireless network, fading and interference are two characteristics of the wireless medium to be dealt with. As an emerging transmit strategy aiming at fading and interference, cooperative communication [2-6] has gained numerous attentions recently. The core idea is that relay nodes can improve the source node's transmission by relaying a replica of its information. With dedicated relay nodes, the hierarchical layering communications can be actualized by cooperative communications, which exploit the broadcast nature and inherent spatial diversity, based on grouping several nodes (each with only one antenna) together into a cluster to form a large transmit or/and receive antenna array. Collaborative clusters are attractive to achieve spatial diversity as well as rate multiplexing by "negotiations" among neighboring nodes with access to more power, better communication capabilities. Cooperation between the nodes fully utilizes rich wireless propagation environments across multiple protocol layers and offers numerous opportunities to improve network performance in terms of throughput [7], reliability $[8,9,10]$, longevity, and flexibility.

The most important element in cooperative communications is coding protocols responsible for interaction between cooperative nodes. In the past few years, several coding strategies have been deployed for cooperative communications. Distributed space-time coding [11] designed for Multiple Input Multiple Output (MIMO) system was introduced; nevertheless synchronizations among cooperative nodes challenge to the space-time coding strategy.

Lately, as the network size grows, traditional relay schemes become increasingly bandwidth inefficient. To break through this bandwidth bottleneck, network coding [12] - a technique originally developed for routing in lossless wireline networks has been recently applied to wireless relay networks. The application of cooperative network coding strategy is based on the fact that network coding has automatically been associated with cooperative communications as it employs intermediate nodes to combine packets. However, the fundamental aspects of wireless communication, interference, fading and mobility make the problem of applying network coding to cooperative communications particular challenging.

This work was supported in part by National Basic Research Program of China (NO.2007CB310601) and National Nature Science Foundation of China (NO.60972021). 


\section{RELATED WORK}

The network coding technology has ramifications across multiple layers. Up to now, two kinds of network coding that enable an unprecedented ability to improve throughput have been proposed: digital network coding (DNC) $[12,13,14,15]$ and analog network coding (ANC) $[16,17,18,19,20,21,22]$.

\section{A. Digital Network Coding $(D N C)$}

DNC is the initial work [12] in this area and is implemented in the network layer or physical-layer but at bit-level, where the relay node combines received bits and forwards them. It is able to realize "max-flow min-cut" capacity in the multicast session in the wireline domain. However, the wireless applications are not merely an extension or simple modification of the wireline case. One should not neglect the characteristics of wireless transmissions, such as inherent broadcast, interference, fading and mobility, when he (she) reuses the vast algorithmic and protocol knowledge established in wireline networks. Therefore, the wireless environment challenges DNC with lossy links. Inspired by its information theoretic scheme and inherent cooperation characteristic, traditional relaying [23] entails loss in spectral efficiency that can be mitigated through network coding in cooperative communications.

Recently developed notion based on joint network coding with channel coding or source coding[24,25,27,28] prompts that network coding is a generalization of source coding and channel coding [14]. From the view of network information theory, it was shown in [26] that joint design of source, channel and network coding in end-to-end transmission could yield much better performance, especially for the situation that source, channel, and network separation between these codes does not hold in underlying networks. Under these circumstances, DNC is proposed to joint with channel coding as cooperative diversity. Hausl [24] studied the unified framework of network coding and channel coding to realize the optimal end-to-end communication. In his work, LDPC and Turbo codes are used as surrogate to fulfill the job. BAO [25] proposed a joint channel-network coding matched network topology, known as GANCC (Generalized adaptive network coded cooperation), considering time-variant feature of wireless mobility. Furthermore, Li [29] adopted partial factor graphs instead of the whole system factor graph to achieve practical design for LDPC in the single user relay scenario.

\section{B. Analog Network Coding(ANC)}

Analog network coding (ANC, also known as physicallayer network coding, PNC), which employs the wireless broadcast nature and the idea of diversity at the signal level, was proposed as the counterpart of DNC. Network coding reflects the essence of cooperation in the context of networks, especially in the two-way relay channel (TRC), where two sources desire to exchange information with the help of one relay in between. Indeed, a vast portion of the literature on $\mathrm{ANC}$, especially in the realm of information theory, has been devoted to TRC [16-22] and its development to an adequate extent.

In this regard, Zhang et al. [21] first shed light on PNC; their work dealt with EM signal reception and modulation by self-defined mappings of the mixed signals. However, the proposed ANC mapping scheme requires strict synchronizations among the senders at the level of symbol, carrier frequency and carrier phase, which will consume significant resources in practice. Another seminal work in this area was presented as analog network coding (ANC) [16], where algebraic relationships among the mixed signals, whose amplitude and phase have been distorted in transmission, were derived. As one of the signals is already known to the users in $\mathrm{TRC}$, they can solve for the other desired signal by these algebraic relationships. Nevertheless, ANC and its algorithm cannot be extended to more general channel models except TRC and also vulnerable to channel fading effects. Furthermore, the work in [15] proposes a strategy of XOR-bit combination in TRC, where the relay node decodes the information from the users and then carries out the XOR operation to the decoded bits before broadcasting them. After another XOR operation in the local receivers, the two users are able to obtain the information. The whole scheme is in the network layer, and the physical layer strategy is not considered explicitly.

Oechtering [18] and Popovski [19] analyzed the capacity of TRC using PNC but without the detailed coding and decoding strategies. Other reports on PNC were devoted to its various extensions, such as that by Xue[20] and Narayanan[17], who studied the joint design of ANC with other coding strategies to approach optimal rates as viewed from a network-wide perspective. The work in [22] attempted to explore PNC in a unicast session due to its salient results in multicast scenarios.

In this paper, these two kinds of network coding way in wireless cooperative communications are developed. For DNC, the problem of network coding in a lossy link, which leads to a fading effect, is dealt with; and for ANC, the problem of two signals meeting at a common node, which leads to interference effect, is dealt with. Both two network coding way are in the frame of MARC cooperation, employing a extra relay node to perform network coding functions.

\section{SYSTEM MODEL}

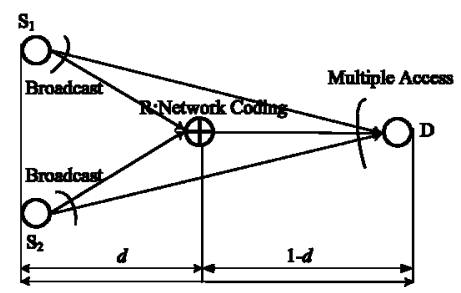

Figure 1. MARC model.

The future distributed wireless network such as sensor network is generally built as a hierarchical structure by placing a sparse network of access points connected by a highbandwidth network within a random homogeneous ad hoc network, in which wireless relay nodes serve exclusively as forwarders [30]. In addition, the hierarchical sensor network with an access point and a single forwarding node can be modeled as a Multiple Access Relay Channel (MARC), which is a multi-source extension of the well-known single-user relay channel [30]. Basically, a MARC consists of two Sources $\left(\mathrm{S}_{1}\right.$, 
$\mathrm{S}_{2}$ ), one Relay (R), and one Destination (D), as in Fig.1. This MARC model has a symmetric positioning of $S_{1}, S_{2}$ with respect to $R$ and $D$. The four nodes are collinear with both $S_{1}$ and $S_{2}$ at the origin, and $D$ is a unit distance away from the origin. The relay moves along the line connecting $\mathrm{D}$ with the origin. The distance between $\mathrm{S}$ and $\mathrm{R}$ is set to $d$. Path loss is proportional to $1 / d^{2}$. The channels between each node are independent of each other. Perfect global channel knowledge is assumed at all nodes. Since radio terminals cannot transmit and receive simultaneously in the same frequency band, most cooperative strategies are based on half-duplex mode. The nodes are allocated orthogonal channels as TDMA reasonable to low cost requirements, and synchronization of TDMA is in packets level. $S_{1}$ and $S_{2}$ are assumed to send message with no priority. One block transmission is separate into two consecutive time slots, normalized to $t_{1}+t_{2}=1$. Furthermore, Source codeword length for one block is $N$ (for brief and to the point, the symbols of $S_{1}, S_{2}$ are equal and independent with each other), also split into two sub-blocks, each with $t_{1} N, t_{2} N$ long codewords accordingly. the baseband-equivalent, discretetime channel model is,

$$
y_{j}=h_{i j} x_{i}+z_{i j}
$$

where $x_{i}$ denotes an input signal from node $i ; y_{i}$ denotes an output signal from node $j$. Besides, lowercase is for the signals and uppercase is for the messages included in the signals, such as $x_{i}, y_{i}$ represent signals and $X_{i}, Y_{i}$ represent messages in them; $h_{i j}$ denotes a channel coefficient from node $j$ to node $i$, reflecting the effects of path-loss and Rayleigh fading. $z_{i j}$ represents an additive noise and other interference in the system.

\section{NeTWORK-CODED COOPERATIVE PROTOCOL}

The proposed cooperative protocol is based on network coding, which functions broadly across each layer in network protocol stack. This article illustrates two popular ways of combining information: DNC and ANC. DNC, a modulo-sum of information contents at the bit-level is performed; while ANC, the mixing signals in the free space at the signal-level are used as illustrations. Fig.2 shows the two transmission stages.

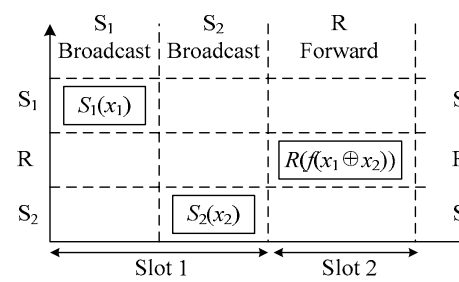

(a) Bit-level modulo-sum

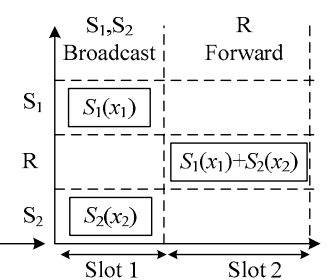

(B) Signal-level mixing.
Figure 2. Network-coded cooperative protocols, (a) DNC bit-level modulosum, (b) ANC signal-level mixing.

\section{A. DNC Cooperative Strategy}

In this mode, the protocol is rooted from decode-andforward. Source nodes $\mathrm{S}_{1}$ and $\mathrm{S}_{2}$ are independent of each other and $\mathrm{R}$ is one of the relevant intermediate nodes. At R, two packets from $S_{1}$ and $S_{2}$ are separately decoded and combined with partial messages in an exclusive OR operation, as shown in Fig.2(a), then forwarded to D. Network coding procedure is accomplished in the sense of cooperation between the source nodes and the relay node. The detailed strategy is as follows, also shown in Fig.3.

\section{1) The first time slot $t_{1}$}

Each source broadcasts codewords $\mathrm{C}_{S_{1}}$ and $\mathrm{C}_{S_{2}}$ to $\mathrm{R}$ and $\mathrm{D}$. $\mathrm{S}_{1}$ and $\mathrm{S}_{2}$ encode their own message $\boldsymbol{X}_{\mathbf{1}}$ and $\boldsymbol{X}_{\mathbf{2}}$ locally, by its own codeword book to $\mathbf{C}_{S 1}$ and $\mathbf{C}_{S 2}$, at the rate of

$$
R_{s 1 r}+R_{s 2 r}=I\left(X_{1}, X_{2} ; Y_{r}\right)
$$

$\mathrm{D}$ receives and stores the data for decoding at the end of the block transmission. The baseband-equivalent discrete-time channel models at $\mathrm{R}$ and $\mathrm{D}$ are separately defined as

$$
\begin{gathered}
y_{r}=\left[\begin{array}{l}
y_{r 11} \\
y_{r 12}
\end{array}\right]=\left[\begin{array}{cc}
h_{s 1 r} & 0 \\
0 & h_{s 2 r}
\end{array}\right]\left[\begin{array}{l}
x_{1} \\
x_{2}
\end{array}\right]+\left[\begin{array}{l}
z_{r 1} \\
z_{r 2}
\end{array}\right] \\
y_{d 1}=\left[\begin{array}{l}
y_{d 11} \\
y_{d 12}
\end{array}\right]=\left[\begin{array}{cc}
h_{s 1 d} & 0 \\
0 & h_{s 2 d}
\end{array}\right]\left[\begin{array}{l}
x_{1} \\
x_{2}
\end{array}\right]+\left[\begin{array}{l}
z_{d 1} \\
z_{d 2}
\end{array}\right]
\end{gathered}
$$

In order to obtain the maximum throughput, sources broadcast at the rates (2), and R is able to decode $\boldsymbol{X}_{\mathbf{1}}$ and $\boldsymbol{X}_{\mathbf{2}}$ with an arbitrarily low error probability. Of course, D would also receive the same messages in the first slot, but it cannot uniquely decode $\boldsymbol{X}_{1}$ and $\boldsymbol{X}_{2}$. The reason lies in that from Fig.1, the physical channel from $\mathrm{S}$ to $\mathrm{D}$ is much more damaged than that from $\mathrm{S}$ to $\mathrm{R}$; generally the following expression is tenable:

$$
I\left(X_{1}, X_{2} ; Y_{r}\right)>I\left(X_{1}, X_{2} ; Y_{d 1}\right)
$$

2) The second time slot $t_{2}$

D needs extra bits

$$
\mathrm{t}_{1} N \cdot\left[I\left(X_{1}, X_{2} ; Y_{r}\right)-I\left(X_{1}, X_{2} ; Y_{d 1}\right)\right]
$$

to decode the message successfully. Thus, the design is to let $\mathrm{R}$ send these extra bits to $\mathrm{D}$ at the rate of

$$
\begin{gathered}
R_{r d}=\mathrm{t}_{1} N \cdot\left[I\left(X_{1}, X_{2} ; Y_{r}\right)-I\left(X_{1}, X_{2} ; Y_{d 1}\right)\right] / \mathrm{t}_{2} N \\
=\frac{\mathrm{t}_{1}}{\mathrm{t}_{2}}\left[I\left(X_{1}, X_{2} ; Y_{r}\right)-I\left(X_{1}, X_{2} ; Y_{d 1}\right)\right] \\
y_{d 2}=h_{r d} x_{3}+z_{d 2}
\end{gathered}
$$

Specifically, R, after decoding the codewords from $\mathrm{S}_{1}$ and $\mathrm{S}_{2}$, estimates $\mathrm{C}_{S 1}$ and $\mathrm{C}_{S 2}$, which then together coded by network codes to generate $k_{\text {net }}$ check bits.

$$
k_{\text {net }}=\mathrm{t}_{1} N \cdot\left[I\left(X_{1}, X_{2} ; Y_{r}\right)-I\left(X_{1}, X_{2} ; Y_{d 1}\right)\right]
$$

The above operation cooperatively performed by the source nodes and the relay node obeys the rule of coding with side information or binning procedure. Furthermore, binning is implemented by extra parity network coding bits (or syndromes). Relay's message, $\boldsymbol{X}_{\mathbf{3}}$, the extra check bits generated based on sources' message, helps $D$ to decode $X_{1}$ and $\boldsymbol{X}_{\mathbf{2}}$ (the elements of $\boldsymbol{X}_{\mathbf{1}}$ and $\boldsymbol{X}_{\mathbf{2}}$ belong to the set $\left.\left\{1,2, \ldots, 2^{\mathrm{t}_{1} N \cdot I\left(X_{1}, X_{2} ; Y_{r}\right)}\right\}\right)$ by restricting them to $2^{\left.\mathrm{t}_{1} N \cdot I\left(X_{1}, X_{2} ; Y_{11}\right)\right)}$ 
bins with the size of $2^{\mathrm{t}_{1} N \cdot\left[I\left(X_{1}, X_{2} ; Y_{r}\right)-I\left(X_{1}, X_{2} ; Y_{d 1}\right)\right]}$ each. Moreover, the "binning" of $\mathrm{R}$ is a random set of bins' indexes to partition the sources' message space thus enlarging the distance of the codewords to make the source's message decodable.

Consequently, the network-coded bits provide extra check bits for decoding in $\mathrm{D}$. This is a design approach by joining channel coding in $S_{1}$ and $S_{2}$ with network coding in $R$, which proves very effective in suppressing noise and fading at the least cost of bandwidth. To some extent, the decode-andforward protocol here can be regarded as a joint routing of parity check bits.

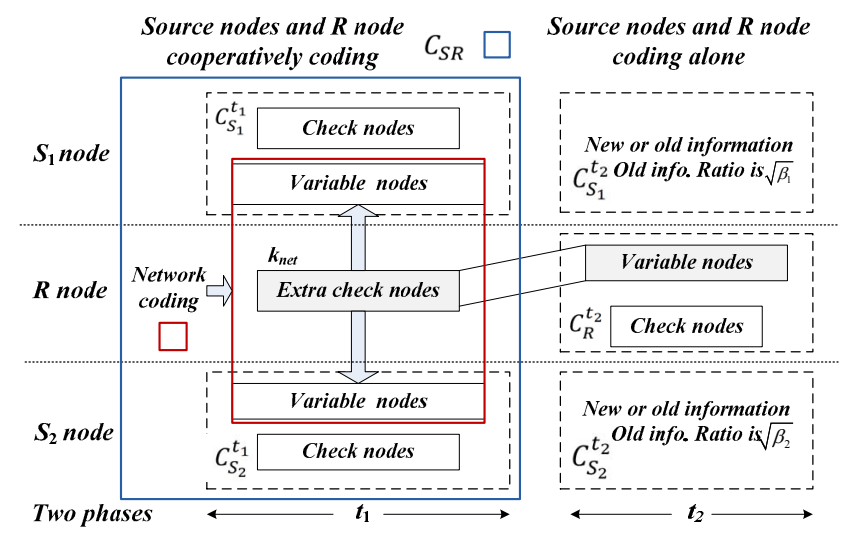

Figure 3. The DNC cooperative strategy.

\section{B. ANC Cooperative Strategy}

In the network-layer network coding protocol, the multiple users each employ one time slot to transmit signals one after another to avoid interference, which is known as orthogonal transmission. Differently, in another popular Analog Network Coding (ANC), the users send signals simultaneously (see Fig.2(b)) without orthogonal scheduling, embracing interference in an innovative way. In the following, $\mathrm{ANC}$ is applied to MARC model and a detailed math model of the protocol is shown.

\section{1) The first time slot $t_{1}$}

By the very broadcast nature of communication in the wireless channel, source nodes $S_{1}$ and $S_{2}$ send out signals simultaneously and both $R$ and $D$ receive mixed signals of $S_{1}$ and $\mathrm{S}_{2}$ in free space. $\mathrm{R}$ receives a signal $y_{r}$, mixed with an additive noise $z_{r}$ :

$$
y_{r}=h_{s 1 r} x_{1}+h_{s 2 r} x_{2}+z_{r}
$$

$\mathrm{D}$ also receives a signal $y_{d 1}$, mixed with another noise $z_{d 1}$ :

$$
y_{d 1}=h_{s 1 d} x_{1}+h_{s 2 d} x_{2}+z_{d 1}
$$

Because signal-level mixing way embraces two signals from $\mathrm{S}_{1}$ and $\mathrm{S}_{2}$ sent at the same time, $y_{r}$ and $y_{d 1}$ become one signal each after mixing in node $\mathrm{R}$ and $\mathrm{D}$.

\section{2) The second time slot $t_{2}$}

$\mathrm{R}$ retransmits and forwards the mixed signals received in the first time slot to the destination $\mathrm{D}$. $\mathrm{R}$ amplifies the received signals, turning into $y_{r}$ and then forwards $y_{r}$ to $\mathrm{D}$, which acquires another sample of the mixed signals of $S_{1}$ and $S_{2}$ to disentangle the signals. Then $\mathrm{D}$ receives a signal $y_{d 2}$ as follows:

$$
\begin{aligned}
& y_{d 2}=h_{r d} y_{r}+z_{d 2} \\
& =h_{r d} h_{s 1 r} x_{1}+h_{r d} h_{s 2 r} x_{2}+h_{r d} z_{r}+z_{d 2}
\end{aligned}
$$

By arranging (10)-(12), a matrix denoted by $\mathbf{H}$ is formed to express the relationship between the source signal matrix $\mathbf{X}$ at $\mathrm{S}_{1}$ and $\mathrm{S}_{2}$ and the output signal matrix $\boldsymbol{Y}$ at D. Accordingly, the equation $\mathbf{Y}=\mathbf{H X}+\mathbf{Z}$ can be easily acquired, i.e.,

$$
\left[\begin{array}{l}
y_{d 1} \\
y_{d 2}
\end{array}\right]=\left[\begin{array}{cc}
h_{s 1 d} & h_{s 2 d} \\
h_{r d} h_{s 1 r} & h_{r d} h_{s 2 r}
\end{array}\right]\left[\begin{array}{l}
x_{1} \\
x_{2}
\end{array}\right]+\left[\begin{array}{c}
z_{d 1} \\
h_{r d} z_{r}+z_{d 2}
\end{array}\right]
$$

Decoding of ANC is to disentangle the mixed signal $\boldsymbol{Y}$ by searching for the maximum independent components with an improved joint approximate diagonalization of eigen-matrices (ARD) algorithm [31] on the assumption that signals are independent of each other. Generally, this holds true in most cases, for each encoder has independent code books. ARD finds a "separate matrix" $\mathbf{V}$ matched to the observed signals $\mathbf{Y}$. Then, the channel coefficients $\mathbf{H}$ and original signals $\mathbf{X}$ can both be recovered with $\mathbf{V}$, as in Fig.4.

In this cooperation, $\mathrm{R}$ only amplifies and forwards the signal $y_{r}$, so the cooperative protocol is a simple amplify-andforwarding (AF). The AF protocol is for the capacity analysis of ANC later. In addition, the number of observed samples at D is very important in ARD. Because we do not make any assumptions of original signal structure, the dimensions of the observed signals $\mathbf{Y}$ must be larger than the dimensions of the original signals $\mathbf{X}$, a necessary condition in BSS. In the ANC model, this condition is satisfied naturally, because two-slot transmissions produce two replicas, $y_{d 1}, y_{d 2}$ of the observed signals at D.

By making use of the blind signal separation method, the requirements for senders' synchronization and channel state information can be removed, which is very attractive, for synchronization is always bothering and tedious in wireless communications.

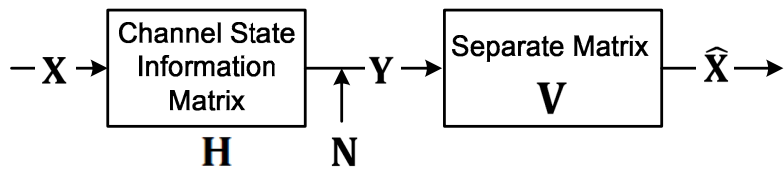

Figure 4. The ANC process in wireless fading channel.

\section{PERFORMANCE OF NETWORK CODED COOPERATIVE PROTOCOLS}

In this section, the capacity of DNC and ANC is evaluated by the mutual information expressions. And BER curves in the Rayleigh fading environment are plotted to give a coarse demonstration that these two network coded cooperative protocols are able to attain the improvements in terms of capacity and reliability. 


\section{A. DNC Capacity}

$\mathrm{DNC}$ is based on the decode-and-forward protocol, in which the transmit rate of $\mathrm{S}_{1}$ and $\mathrm{S}_{2}$ must ensure that $\mathrm{R}$ decodes $x_{1}, x_{2}$ successfully. The throughput of decode-and-forward protocol can be derived from the "max-flow min-cut" theorem. And the DNC based cooperative protocol realizes the "cut-set" bound determined by the link between source nodes and the relay node. Thus, the capacity of DNC is

$$
\begin{gathered}
I_{D N C}\left(X_{1}, X_{2} ; Y_{d}\right)=\min \left\{C_{s r}, C_{s d}+C_{r d}\right\}=I\left(X_{1}, X_{2} ; Y_{r}\right) \\
I_{D N C}\left(X_{1}, X_{2} ; Y_{d}\right)=\frac{1}{4} \log \left[\begin{array}{c}
1+\frac{\gamma}{d^{2}\left(\left|\alpha_{s 1 r}\right|^{2}+\left|\alpha_{s 2 r}\right|^{2}\right)} \\
+2 \gamma^{2} / d^{4}\left|\alpha_{s 1 r}\right|^{2}\left|\alpha_{s 2 r}\right|^{2}
\end{array}\right]
\end{gathered}
$$

$\gamma$ is SNR without fading, $\gamma=P /\left(W \sigma_{0}^{2}\right), \alpha_{i j}$ captures the Rayleigh fading factor involved in channel factor $h_{i j}$.

\section{B. ANC Capacity}

In contrast, multiple users send signals simultaneously in the ANC protocol. According to Fig.2(b), intuitively, the ANC protocol is capable of reducing the number of time slots from 3 to 2 when delivering the same amount of information. Clearly, the ANC protocol is able to improve bandwidth utilization potentially. Since the ANC is based on the amplify-andforward protocol, the achievable mutual information can be written as

$$
I_{P N C}\left(X_{1}, X_{2} ; Y_{d}\right)=\frac{1}{2} \log \left(\left|I+\mathbf{H}^{H} \mathbf{Z}^{-1} \mathbf{H}\right|\right)
$$

where $\mathbf{H}$ is the channel matrix and $\mathbf{Z}=\left[\begin{array}{cc}\sigma_{0}^{2} & 0 \\ 0 & \sigma_{0}^{2}\left(1+h_{r d}^{2}\right)\end{array}\right]$ is the covariance matrix of the noise at the receiver.

\section{Simulations}

In this subsection, capacity and BER performance of the above two network coded cooperative protocols are given.

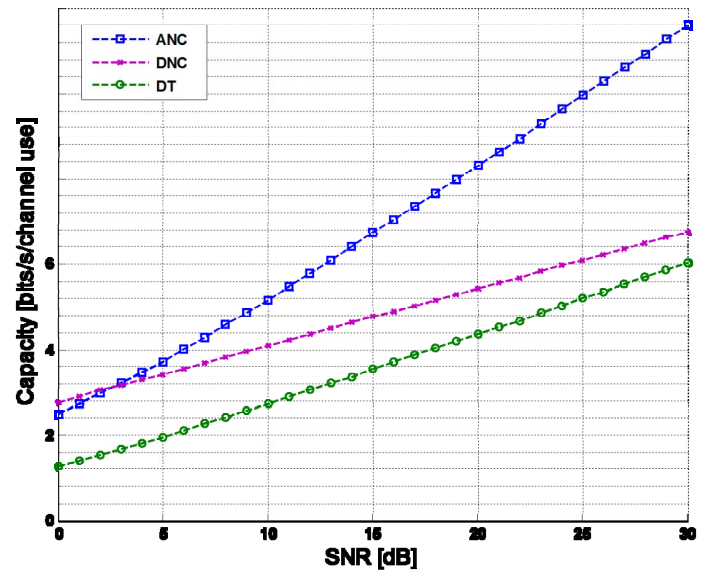

Figure 5. Channel Capacity of APNC, DNC and DT.

(15), (17) and (18) are calculated to evaluate the capacity. Fig.5 gives the capacity of DNC and ANC, with DT plotted as the reference. The ANC strategy obtains more capacity gains because it embraces the interference and employs all the available channels. However, as in the lower SNR region, the ANC capacity is less than the capacity of DNC because the amplify-and-forward strategy also amplifies the noise level.

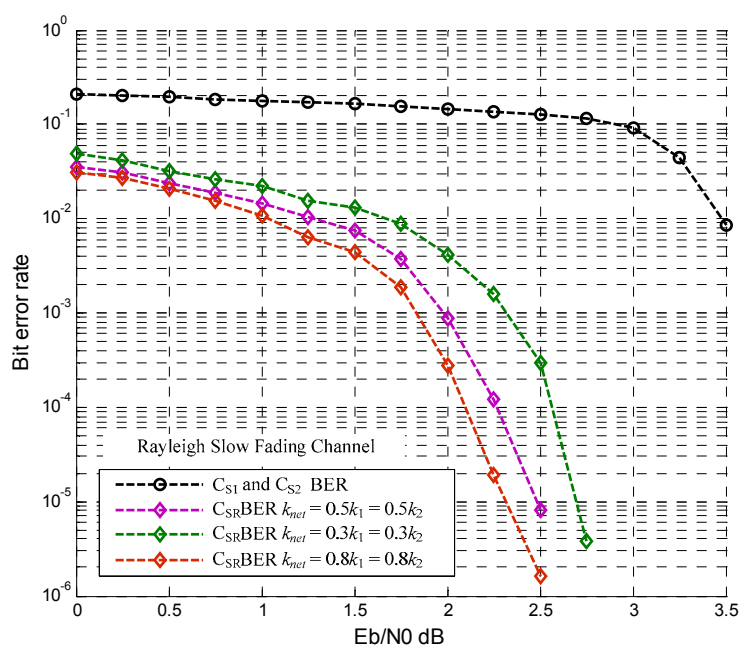

Figure 6. The BER performance under the Rayleigh Slow Fading channel with different lengths of extra check bits

Besides, we also investigate DNC performance of BER with different numbers of extra check bits under Rayleigh Fading channels in Fig.6 and Fig.7. When the extra check bits $k_{\text {net }}=0.5 k_{1}$, the average gain is about $2.2 \mathrm{~dB}$. It is valid that the more extra bits are sent, the better the BER performances are, since the rate of cooperative code is reduced. Thereby, the spatial gain obtained by sending more extra check bits is at the cost of throughput of the whole system.

Then, the BER curves of ANC with 6 different Rayleigh factors $\mathrm{E}\left[\alpha_{i j}^{2}\right]$ are shown in Fig.8. Without any channel equalization or other channel estimation technologies in fading channels, ANC still can perform well in low SNR.

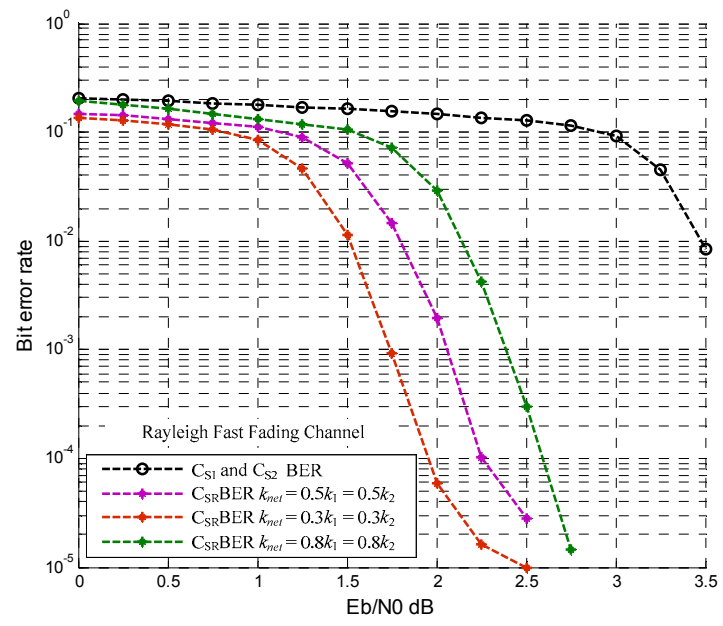

Figure 7. The BER performance under the Rayleigh Fast Fading channel with different lengths of extra check bits 


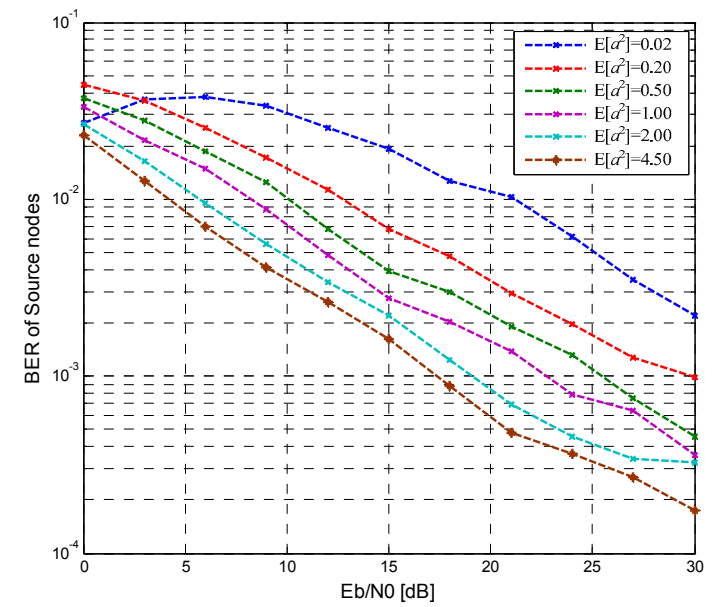

Figure 8. BER of ANC with different Rayleigh fading factor

\section{CONCLUSION}

Network coded cooperative communications for wireless medium presents itself a potentially fruitful technique that would result in remarkable gains both in dealing with the problem fading and interference. Furthermore, two mainstream of network-coded protocols, DNC and ANC, are studied for MARC, which give a vast opportunities to integrate network coding with other transmit technologies, such as joint coding with source, channel coding, or even the compressed sensing technology. And simulations we conducted demonstrate the potential improvements achievable in the throughput and BER performance of the system.

\section{REFERENCE}

[1] F. H.P. Fitzek and M. D. Katz, Cooperation in wireless networks:principles and applications, Springer, Netherlands, 2006.

[2] A. Nosratinia, T. E. Hunter, and A. Hedayat, "Cooperative communication in wireless networks," IEEE Communications Magazine, vol. 42, no. 10, pp.74-80, 2004.

[3] S. E. Erkip and B. Aazhang, "User cooperation diversity, parts I \& II," IEEE Trans. on Communications, vol. 51, no. 11, pp. 1927-1948, Nov. 2003.

[4] J. N. Laneman, D. N. C. Tse and G. W. Wornell, "Cooperative diversity in wireless networks: efficient protocols and outage behavior," IEEE Trans. on Information Theory, vol. 50, no. 12, pp. 3062-3080, Dec. 2004.

[5] J. N. Laneman and G. W. Wornell, "Distributed space-time coded protocols for exploiting cooperative diversity in wireless networks," IEEE Trans. on Information Theory, vol. 49, no. 10, pp. 2415-2425, Oct. 2003.

[6] T. E. Hunter and A. Nosratinia, "Cooperation diversity through coding," in Proceedings of IEEE International Symposium on Information Theory, Lausanne, Switzerland, pp.220, 2002.

[7] G. Kramer, M. Gastpar and P. Gupta, "Cooperative strategies and capacity theorems for relay networks," IEEE Trans. on Information Theory, vol. 51, no.9, pp. 3037-3063, Sep. 2005.

[8] A. Chakrabarti, A. de Baynast, A. Sabharwal and B. Azahang, "Low density parity check codes for the relay channel," IEEE Journal on Selected Areas in Communications, vol. 25, no. 2, pp. 280-291, 2007.

[9] P. Razaghi and W. Yu, "Bilayer LDPC codes for the relay channel," in Proceedings of IEEE International Conference on Communications, Istanbul, vol. 4, pp.1574-1579, Jun. 2006.

[10] M. A. Khojastepour, "Distributed cooperative communications in wireless networks," PhD thesis, Rice University, 2004.
[11] J. N. Laneman and G. W. Wornell, "Distributed space-time coded protocols for exploiting cooperative diversity in wireless networks," IEEE Trans. on Information Theory, vol. 49, no. 10, pp. 2415-2425, Oct. 2003

[12] R. Ahlswede, C. Ning, S.-Y. R. Li and R. W. Yeung, "Network information flow," IEEE Trans. on Information Theory, vol. 46, no. 4, pp. 1204-1216, 2000.

[13] S. Deb, M. Effros, and T. Ho, "Network coding for wireless applications: a brief tutorial," in Proceedings of International Workshop Wireless Adhoc Networks (IWWAN), London, UK, 2005.

[14] R. W. Yeung, S-Y. R. Li and N. Cai, Network coding theory, Foundations and Trends in Communications and Information Theory, Now Publishers Inc, 2006.

[15] S. Katti, H. Rahul and W. Hu, "XORs in the air: practical wireless network coding," IEEE/ACM Tran. on Networking, vol. 16, no.3, pp. 497-510, 2008

[16] S. Katti, S. Gollakota and D. Katabi, "Embracing wireless interference: analog network coding," in Proceedings of ACM SIGCOMM Computer Communication Review 37, pp. 397-408, 2007.

[17] K. Narayanan, M. P. Wilson and A. Sprintson, "Joint physical layer coding and network coding for bi-directional relaying," in Proceedings of 45th Allerton Conference Communication, Control, and Computing, 2007.

[18] J. Oechtering, I. Bjelakovic, C. Schnurr and H. Boche, "Broadcast capacity region of two-phase bidirectional relaying," IEEE Trans. on Information Theory, vol.54, no.1, pp. 454-458, 2008.

[19] P. Popovski, H. Yomo, "Physical network coding in two-way wireless relay channel," in IEEE International Conference Communication (ICC), pp.707-712, 2007.

[20] F. Xue and S. Sandhu, "PHY-layer network coding for broadcast channel with side information," in IEEE Information Theory Workshop (ITW), 2007.

[21] S. Zhang, S. Liew and P. P. K. Lam, "Hot Topic: Physical layer network coding," in Proceedings of ACM MOBICOM, Los Angeles, CA, USA, pp. 358-365, 2006.

[22] H. M. Zimmermann and Y. C. Liang, "Physical layer network coding for uniCast applications," in Proceedings of IEEE Vehicular Technology Conference (VTC), Marina Bay, Singapore, pp. 11-14, 2008.

[23] T. M. Cover and A. A. El Gamal, "Capacity theorems for the relay channel," IEEE Trans. on Information Theory, vol. 25, no. 5, pp. 572584, Sept.1979.

[24] C. Hausl, F. Schreckenbach, I. Oikonomidis and G. Bauch, "Iterative network and channel decoding on a tanner graph," in Proceedings of 43rd Annual Allerton Conference on Communications, Control, and Computing, Sept. 2005.

[25] X. Bao and J. Li, "Matching code-on-graph with networks-on-graph: adaptive network coding for wireless relay networks," in Proceedings of 43rd Annual Allerton Conference on Communications, Control, and Computing, Champaign IL, Sept. 2005.

[26] M. Effros, M. Medard, T. Ho, S. Ray, D. R. Karger and R. Koetter, "Linear network codes: a unified framework for source, channel and network coding," in DIMACS workshop on network information theory, DIMACS Center, Rutgers University, Piscataway, NJ, pp. 197, 2003.

[27] L. Xiao, T. Fuja, J. Kliewer and D. Costello, "A network coding approach to cooperative diversity," IEEE Trans. on Information Theory, vol. 53, no. 10, pp. 3714-3722, Oct. 2007.

[28] Y. Sichao and K. Ralf, "Network coding over a noisy relay: a belief propagation approach," in Proceedings of IEEE International Symposium on Information Theory, pp. 801-804, Jun. 2007.

[29] Li C, Khojastepoury M. A., Yuey G. et al. Performance analysis and code design for cooperative relay channels. In Proc. $40^{\text {th }}$ Annual Conference on Information Sciences and Systems, 2006, 1032-1037.

[30] L. Sankaranarayanan, G. Kramer, and N. B. Mandayam, "Hierarchical sensor networks: capacity bounds and cooperative strategies using the multiple-access relay channel model," in Proceedings of 1st Annual IEEE Communications Society Conference on Sensor and Ad Hoc Communications and Networks (SECON), pp. 191-199, Oct. 2004.

[31] B. DU and Jun Zhang, "A Complex-valued ICA Approach to Physicallayer Network Coding over Wireless Fading Channels," unpublished. 